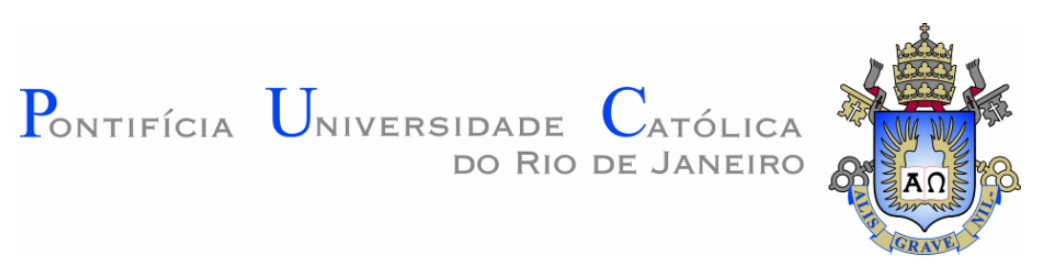

Carolina Leopoldino Thomé

\title{
Soldagem por termofusão aplicada em tubos fabricados de poliamida 12
}

\section{Dissertação de Mestrado}

Dissertação apresentada como requisito parcial para obtenção do título de Mestre pelo Programa de PósGraduação em Engenharia de Materiais e de Processos Químicos e Metalúrgicos da PUC-Rio.

Orientador: Profa. Ivani de Souza Bott Co-orientador: Prof. José Roberto Moraes

D'Almeida 


\section{Carolina Leopoldino Thomé}

\section{Soldagem por termofusão aplicada em tubos fabricados de poliamida 12}

Dissertação apresentada como requisito parcial para obtenção do título de Mestre pelo Programa de PósGraduação em Engenharia de Materiais e de Processos Químicos e Metalúrgicos do departamento de Engenharia Química e de Materiais do Centro Técnico Científico da PUC-Rio. Aprovada pela Comissão Examinadora abaixo assinada.

Profa. Ivani de Souza Bott Orientadora e Presidente Departamento de Engenharia Química e de Materiais - PUC Rio

Prof. José Roberto Moraes D'Almeida Co - orientador Departamento de Engenharia Química e de Materiais - PUC Rio

Prof. Luís Felipe G. de Souza Centro Federal de Educação Tecnológica Celso Suckow da FonsecaCEFET/RJ

Prof. Silvio Romero de Barros Centro Federal de Educação Tecnológica Celso Suckow da FonsecaCEFET/RJ

Prof. Marcio da Silveira Carvalho Coordenador Setorial de Pós-Graduação do Centro Técnico Científico da PUC-Rio

Rio de Janeiro, 15 de Abril de 2016 
Todos os direitos reservados. É proibida a reprodução total ou parcial do trabalho sem autorização da universidade, do autor e do orientador.

\section{Carolina Leopoldino Thomé}

Graduou-se em Engenharia de Produção pela Universidade de Barra Mansa. Ingressou no curso de mestrado em Engenharia de Materiais no ano 2014.

Ficha Catalográfica

Thomé, Carolina Leopoldino
Soldagem por termofusão aplicada em
tubos fabricados de poliamida 12 / Carolina
Leopoldo Thomé ; orientador: Ivani de Souza
Bott ; co-orientador: José Roberto Moraes
D'Almeida. -2016 .
117 f. : il. (color.) ; $30 \mathrm{~cm}$
Dissertação (mestrado)-Pontifícia
Universidade Católica do Rio de Janeiro,
Departamento de Engenharia Química e de
Materiais, 2016.
Inclui bibliografia
1. Engenharia Química - Teses. 2.
Engenharia de materiais - Teses. 3. Soldagem por
termofusão. 4. Configurações de parâmetros. 5.
Tubos de poliamida 12. I. Bott, Ivani de Souza. II.
Almeida, José Roberto Moraes d'. III. Pontifícia
Universidade Católica do Rio de Janeiro.
Departamento de Engenharia Química e de
Materiais. IV. Título.

CDD: 620.11 
Dedico este trabalho às pessoas que sempre torceram pelo meu progresso e sucesso, a todos aqueles que sempre me apoiaram. 


\section{Agradecimentos}

Primeiramente agradeço a Deus e toda a espiritualidade pela força, apoio e direção que sempre tive em todos os caminhos de minha vida. Agradeço por nunca me faltar fé e por acreditar que todos os meus sonhos e desejos podem ser alcançados e, por todos os que eu já conquistei.

À professora Ivani, agradeço com todo o meu carinho por ter aceito meu pedido de orientação e principalmente pela proposta de pesquisa a mim oferecida. Agradeço por todas as dicas, sugestões e conselhos dados durante o desenvolvimento deste projeto.Tenho certeza de que tudo o que me foi dito servirá como auxílio em toda minha carreira profissional.

Ao professor José Roberto D'Almeida pela co-orientação e principalmente pela idéia do tema da pesquisa. Agradeço por todas as dicas e sugestões sobre a caracterização do material estudado.

À aluna de iniciação científica, Thaís Bastos, pela eficiência e apoio durante o projeto, me auxiliando na realização das soldagens, preparação das amostras, ensaios de caracterização e, principalmente por estar sempre disposta e com boa vontade em me ajudar.

Aos meus queridos amigos de Volta Redonda, aos amigos do Rio e aos novos amigos que adquiri durante o mestrado, em especial à Isabella Jorge e ao Reynel Castellanos. Vocês fazem parte de minha história e acompanharam comigo mais uma conquista. Obrigada pelo carinho e consideração.

À minha mãe Ana Paula, minha avó Conceição e todos os meus familiares que estiveram presentes nos momentos em que mais necessitei. À minha tia Luciana que me acolheu em sua casa para que eu pudesse concluir o mestrado. Obrigada a todos vocês por acreditarem que sou capaz!

Ao Centro de Tecnologia Senai Solda. Ao Lincoln e Barreto pela autorização da realização das soldagens. Ao Jorge Wanderlei pela proposta de trabalho com os tubos de Poliamida 12. 
Ao Gilberto Cunha pela disponibilidade na realização dos procedimentos, por todo auxílo, atenção e paciência.

À indústria PolyEasy, pelo fornecimento dos tubos de poliamida 12. Ao Jesus pelo apoio, disposição em esclarecer minhas dúvidas e intermediação com o fornecimento dos tubos.

Ao CEFET-Rio, ao professor Luís Felipe Souza, Hiron Akira e Marcelo Avelelas pelo auxílio com os ensaios de tração.

Ao Departamento de Engenharia Civil da Puc-Rio, professor Flávio Silva e Euclides Neto pelo apoio nos ensaios de rigidez.

Ao professor Roberto de Avillez pela disposição em ajudar na obtenção dos dados de cristalinidade pelos dados de DRX.

Aos professores das disciplinas que cursei, ao pessoal do departamento de administração da pós-graduação do Departamento de Engenharia de Materiais da PucRio.

À Puc-Rio, ao Conselho Nacional de Desenvolvimento Cientifico e Tecnológico (CNPq) pelo financiamento da bolsa recebida. À Coordenação de Aperfeiçoamento de Pessoal de Nível Superior (CAPES) pelo oferecimento da bolsa "Capes taxas escolares". 


\section{Resumo}

Thomé, Carolina Leopoldino; Bott, Ivani de Souza; D`Almeida, José Roberto Moraes. Soldagem por termofusão aplicada em tubos fabricados de poliamida 12. Rio de Janeiro, 2016. 117p. Dissertação de Mestrado Departamento de Engenharia Química e de Materiais, Pontifícia Universidade Católica do Rio de Janeiro.

A utilização de tubos poliméricos, em sistemas de drenagem e instalações de linhas de gás está sendo cada vez mais aplicada devido à diversas características positivas desses materiais. Existem várias aplicações nas quais o material polimérico pode substituir o metálico com vantagem, pois possuem maior flexibilidade (em comparação aos tubos de aço), menor peso (consequente facilidade de manuseio), maior resistência química a diversos fluidos e ao ambiente, resistência a abrasão, baixa densidade, baixa condutividade térmica, não necessitam de pintura, além de possuírem custos menores. Porém, apesar desse tipo de tubo despertar interesse crescente em consequência da possibilidade de diversificação de formas e de suas propriedades atraentes, atualmente diversas indústrias ainda consideram sua utilização como algo não tão vantajoso, optando pela utilização de tubos como os de aço. Algumas das desvantagens dos polímeros levadas em consideração no momento da escolha entre o tipo de material a ser aplicado (comparados ao aço), podem ser: baixa resistência à altas temperaturas, baixa resistência mecânica e alto coeficiente de dilatação térmica. Um ponto crucial para as indústrias que utilizam os tubos poliméricos é o processo de soldagem dos mesmos. A forma mais tradicional de soldagem desses tubos é realizada através do processo por termofusão ou hot plate welding (soldagem por chapa quente), onde os tubos são soldados face a face (solda de topo). O estudo das variações dos parâmetros de soldagem permite que algumas das exigências e requisitos padronizados possam ser reavaliados através de experimentos, de forma a obter melhor compreensão ou até mesmo a otimização dos resultados gerados. Os procedimentos que foram utilizados na execução das soldas a 
serem analisadas neste trabalho são embasados na norma alemã DVS 2207, sendo o padrão mais utilizado atualmente nas indústrias como base para a aplicação dos parâmetros e requisitos do processo. Apesar desse processo oferecer grande segurança e confiabilidade, a divulgação na literatura ainda é limitada e algumas questões precisam ser discutidas, como por exemplo, a eficácia das faixas de trabalho pré-definidas. Este trabalho tem por objetivo identificar as combinações de parâmetros que permitam gerar juntas soldadas de tubos de poliamida 12 que possuam melhor qualidade e consequentemente bom desempenho. As juntas foram realizadas no Centro de Tecnologia Senai Solda (CTS). Foram estudadas diferentes configurações dos parâmetros de processo, variando pressão de preaquecimento, pressão de aquecimento e temperatura da chapa. Mudanças como, a variação da massa e a degradação do material, influenciadas por tais parâmetros, foram analisadas através dos ensaios de análise termogravimétrica. As respostas mecânicas foram analisadas por testes de rigidez e tração.

\section{Palavras-chave}

Soldagem por termofusão; configurações de parâmetros; tubos de Poliamida 12. 


\section{Abstract}

Leopoldino Thomé, Carolina; Bott, Ivani de Souza (Advisor); D’Almeida, José Roberto Moraes (Co-Advisor). Hot Plate Welding applied to manufactured tubes of Polyamide 12. Rio de Janeiro, 2016. 117p. MSc. Dissertation - Departamento de Engenharia Química e de Materiais, Pontifícia Universidade Católica do Rio de Janeiro.

The use of polymeric pipes in drainage systems and gas lines facilities are increasingly being applied due to several positive characteristics of these materials. There are several applications in which the polymeric material can replace metallic advantageously because they have greater flexibility (compared to steel pipes), lower weight (consequent ease of handling), increased chemical resistance to various fluids and to the environment, abrasion resistance, low density, low thermal conductivity, doesn't require painting, in addition to having lower costs. However, despite of such tube are awakening growing interest as a result of the possibility of diversification of forms and their attractive properties, currently several industries still consider it as something not so advantageous, opting for the use of pipes as the steel. Some of the disadvantages of the polymers taken into account in the choice of the type of material to be applied (compared to steel) can be: low resistance to high temperatures, low mechanical strength and high thermal expansion coefficient. A crucial point for the industries that use polymer pipes is their welding process. The most traditional way welding of these pipes is performed through the process by thermal fusion. It's called "hot plate welding", where the pipes are welded face to face (butt-weld). The study of changes of welding parameters allows some of the requirements and standards can be reviewed through experiments in order to obtain a better understanding or even optimizing the results generated. The procedures used in the execution of the joints analyzed in this work are grounded in the German standard - DVS 2207. It's the most used standard in the industries as a basis for the application of parameters and process requirements. Although this process offer great safety and reliability, the disclosure in the literature is still limited and some issues need to be discussed, such as the 
effectiveness of pre-defined working range. This work aims to identify the combinations of parameters to generate welded joints of 12 polyamide pipes that have better quality and therefore good performance. The joints were made in the Centro de Tecnologia Senai - Solda (CTS). Different settings of processing parameters were studied. The microstructural changes like union of surfaces, mass variation and degradation of the material, were analyzed by the thermogravimetric analysis tests and the macro-structural changes (mechanical response) to the influence of these parameters were analyzed by stiffness and tensile tests.

\section{Keywords}

Termofusion Welding; parameters settings; polyamide12 pipes. 


\section{Sumário}

1. Introdução 23

1.1. Objetivo 24

1.2. Objetivos específicos $\quad 24$

2. Revisão Bibliográfica 26

2.1. Características gerais dos polímeros 26

2.1.1. Cristalização dos polímeros semicristalinos 27

$\begin{array}{ll}\text { 2.1.2. Cristalinidade } & 28\end{array}$

2.1.3. Micélio com borda $\quad 29$

2.1.4. Densidade 30

2.2. Comportamento térmico dos polímeros 31

2.2.1. Temperatura de transição vítrea $(\mathrm{tg})$

2.2.2. Temperatura de fusão $(\mathrm{Tm})$

2.2.3. Temperatura de cristalização (TC) 32

2.2.4. Temperatura de degradação 32

2.3. Propriedades mecânicas dos polímeros 33

2.4. Influência dos parâmetros de processo na poliamida 12

2.4.1. Efeito da temperatura 35

2.5. Características gerais da poliamida $12 \quad 37$

2.5.1. Estrutura molecular da poliamida $12 \quad 37$

2.6. Soldagem em materiais poliméricos 38

2.6.1. Soldagem de poliamida $12 \quad 39$ 
2.6.2. Principais parâmetros do processo de soldagem por termofusão $\quad 40$

2.6.2.1. Influência das tensões residuais e alinhamento molecular $\quad 40$

2.6.3. Influência da largura da zona termicamente afetada 42

2.7. Processo de soldagem por termofusão ou chapa quente (hot plate welding) 42

2.7.1. Tipos de soldagem por chapa quente 43

2.7.2. Soldagem por contato 44

2.7.2.1. Chapas adequadas à soldagem por contato 44

2.7.2.2. Soldagem por contato através de baixas temperaturas 44

2.7.2.3. Soldagem por contato através de altas temperaturas 44

2.7.3. Máquinas e equipamentos utilizados na soldagem por termofusão 45

2.7.4. Descrição técnica do processo de soldagem por termofusão 46

2.7.4.1. Sequência de operações de máquinas horizontais manuais $\quad 46$

2.7.5. Parâmetros de processo 49

2.7.5.1. Temperatura da chapa quente 50

$\begin{array}{ll}\text { 2.7.5.2. Pressão de arraste } & 51\end{array}$

2.7.5.3. Pressão de preaquecimento (P1) 51

2.7.5.4. Pressão de aquecimento /fusão (P2) 51

2.7.5.5. Pressão de união (P3) 51

2.7.5.6. Tempo de resfriamento 52

2.7.5.7. Processo de união de tubos de poliamida 11 e 12

2.7.6. Utilização da poliamida 12 em tubulações de gás natural 56

2.7.6.1. Especificações técnicas das tubulações utilizadas 57

2.8. Métodos de caracterização 57

2.8.1. Análise termogravimétrica (TGA) 57 
2.8.2. Calorimetria diferencial de varredura (DSC) 59

2.8.2.1. Propriedades importantes para o processo de soldagem 60

$\begin{array}{ll}\text { 2.8.2.1.1. Calor de fusão } & 60\end{array}$

2.8.3. Ensaio de tração 61

2.8.3.1. Propriedades de tração em polímeros semicristalinos 63

2.8.4. Ensaio de ensaio de rigidez 63

2.8.5. Difração de Raios X (DRX) 64

3. Materiais e Métodos 66

3.1. Material Utilizado 66

3.2. Procedimentos Experimentais 67

$\begin{array}{ll}\text { 3.2.1. Preparação dos tubos } & 67\end{array}$

$\begin{array}{ll}\text { 3.2.2. Soldagem } & 67\end{array}$

3.3. Ensaios de Tração 72

3.3.1. Preparação dos Corpos de Prova 72

$\begin{array}{ll}\text { 3.3.2. Procedimento de Teste } & 73\end{array}$

3.4. Ensaios de rigidez $\quad 75$

3.4.1. Preparação dos Corpos de Prova 75

3.4.2. Procedimento de Teste 75

3.5. Ensaios de Calorimetria Diferencial de Varredura (DSC) 76

3.5.1. Preparação das Amostras 76

$\begin{array}{ll}\text { 3.5.2. Procedimento de Teste } & 77\end{array}$

3.6. Ensaios de Análise Termogravimétrica (TG) 77

$\begin{array}{ll}\text { 3.6.1. Procedimento de Teste } & 77\end{array}$ 
3.7. Ensaios de Difração de Raios X

$\begin{array}{ll}\text { 3.7.1. Preparação das Amostras } & 78\end{array}$

$\begin{array}{ll}\text { 3.7.2. Procedimento de Teste } & 78\end{array}$

4. Resultados Experimentais $\quad 79$

4.1. Ensaio de tração $\quad 79$

4.1.1. Influências dos parâmetros de soldagem no limite de resistência 82

4.1.1.1. Influência da temperatura de soldagem 82

4.1.1.2. Influência do tipo de resfriamento 83

4.1.1.3. Influência do tempo de resfriamento com pressão 84

4.2. Ensaio de Rigidez 85

4.2.1. Influências dos parâmetros de soldagem na rigidez 86

4.2.1.1. Influência da temperatura de soldagem 86

4.2.1.2. Influência do tipo de resfriamento 88

4.2.1.3. Influência do tempo de resfriamento com pressão 89

4.3. Calorimetria Diferencial de Varredura (DSC) 90

4.4. Análise Termogravimétrica (TGA) 95

4.4.1. Ensaios das juntas soldadas 95

4.4.2. Ensaios das rebarbas 98

4.5. Ensaio de difração de raios $x \quad 100$

5. Discussão dos resultados 106

5.1. Ensaio de tração 106

5.2. Ensaio de Rigidez 107

5.2.1. Influência da temperatura de soldagem 108 
5.2.2. Influência do tipo de resfriamento

5.3. Influência do tempo de resfriamento com pressão

5.4. Sobre a cristalinidade da Poliamida 12

6. Conclusões 


\section{Lista de Figuras}

Figura 2-1 - Esferulitos observados através de microscópio óptico de luz polarizada [6]

Figura 2-2 - Estrutura esferulítica de materiais poliméricos [4].

Figura 2-3 - Modelo do micélio com borda para um polímero semi cristalino, indicandas regiões cristalinas e amorfas [4].

Figura 2-4 - Diagrama deformação X tempo [22].

Figura 2-5 - Gráfico de tensão versus deformação da Poliamida 12, sob diferentes temperaturas [11].

Figura 2-6 - Fórmula estrutural completa (a) e condensada linear (b) para a Poliamida 12 [3].

Figura 2-7 - União de interfaces poliméricas a partir da difusão das cadeias moleculares [8].

Figura 2-8 - Variação da taxa de cisalhamento com variação apenas da espessura do líquido cisalhado [14].

Figura 2-9 - Resumo do processo de soldagem por termofusão [20] [21]. 43

Figura 2-10 - Máquina de solda por termofusão - tipo horizontal [23]. $\quad 45$

Figura 2-11 - Máquina de solda por termofusão - tipo vertical [23]. $\quad 46$

Figura 2-12 -Etapas do processo de soldagem de topo através de máquina manual do tipo horizontal [24].

Figura 2-13 - Casquilhos de acordo com o diâmetro do tubo utilizado [25] 47 
Figura 2-14 - Diagrama dos parâmetros de soldagem, sem dupla pressão e sem dupla união [20].

Figura 2-15 - Esquema ilustrativo dos parâmetros de soldagem por termofusão [27].

Figura 2-16 - Curva TG para a oxidação do grafite [5]. 58

Figura 2-17 - Termograma da análise DSC [35]. 59

Figura 2-18 - Termogramas da Poliamida 12 [38]. 60

Figura 2-19 - Teste de tração realizado em amostras de VESTAMID NRG, PP E PEAD [9]. $\quad 62$

Figura 2-20 - Estágios do processo de deformação por esforços de tração em polímeros semicristalinos [46].

Figura 3-1 - Equipamento utilizado nos processos de soldagem. 68

Figura 3-2 - Tubos de PA12 soldados por termofusão. 69

Figura 3-3 - Formato do corpo de prova para teste de tração [44]. 72

Figura 3-4 - (a) Corpos-de-prova com rebarba (b) Corpos-de-prova sem rebarba

Figura 3-5 - Máquina de ensaio universal - modelo INSTRON 598.

Figura 3-6 - Formato do corpo de prova para teste de rigidez.

Figura 3-7 - Equipamento utilizado para os ensaios de rigidez. 76

Figura 3-8 - Analisador Térmico simultâneo DSC/TG usado na pesquisa. 77

Figura 3-9 - Difratômetro Bruker D8 Discover usado neste trabalho. 79

Figura 4-1 - Limite de resistência médio entre os corpos-de-prova com e sem rebarba de todas as condições estudadas.

Figura 4-2 - Limite de resistência médio entre os corpos-de-prova com e sem rebarba dos conjuntos 1, 2, 3 e 4 . 
Figura 4-3 - Limite de resistência médio entre os corpos-de-prova com e sem rebarba dos conjuntos 5 e 6 .

Figura 4-4 - a) Limite de resistência médio entre os corpos-de-prova com rebarba dos conjuntos 1 e 5. b) Limite de resistência médio entre os corpos-de-prova sem rebarba.

Figura 4-5 - Limite de resistência médio entre os corpos-de-prova com e sem rebarba dos conjuntos 2 e 6 .

Figura 4-6 - Limite de resistência médio entre os corpos-de-prova com e sem rebarba dos conjuntos 3 e 5 soldados a $220^{\circ} \mathrm{C}$.

Figura 4-7 - Limite de resistência médio entre os corpos-de-prova com e sem rebarba dos conjuntos 4 e 6 soldados a $240^{\circ} \mathrm{C}$.

Figura 4-8 - Curvas de carregamento X deslocamento axial obtidas através dos ensaios de rigidez.

Figura 4-9 - Rigidez média dos tubos soldados - comparação entre os conjuntos 1 e 2 .

Figura 4-10 - Rigidez média dos tubos soldados - comparação entre os conjuntos 3 e 4 .

Figura 4-11 - Rigidez média dos tubos soldados - comparação entre os conjuntos 5 e 6 .

Figura 4-12 - Rigidez média dos tubos soldados - comparação entre os conjuntos 1 e 5 soldados a $220^{\circ} \mathrm{C}$.

Figura 4-13 - Rigidez média dos tubos soldados - comparação entre os conjuntos 2 e 6 , soldados a $240^{\circ} \mathrm{C}$.

Figura 4-14 - Rigidez média dos tubos soldados - comparação entre os conjuntos 4 e 6 a $240^{\circ} \mathrm{C}$. 
Figura 4-15 - Rigidez média dos tubos soldados - comparação entre os conjuntos 3 e 5 a $220^{\circ} \mathrm{C}$.

Figura 4-16 - Curva DSC da amostra do tubo de PA12 conforme recebido.

Figura 4-17 - Curvas DSC das amostras das juntas soldadas. 92

Figura 4-18 - Curvas DSC das rebarbas da juntas soldadas. 93

Figura 4-19 - Curva TG para amostra do tubo de poliamida 12 na condição de conforme recebido. 96

$\begin{array}{ll}\text { Figura 4-20- Curvas TG das juntas soldadas. } & 97\end{array}$

Figura 4-21- Curvas TG das rebarbas das amostras soldadas. 99

Figura 4-22-Difratograma da amostra do tubo de poliamida 12 (conforme recebido).

Figura 4-23- Difratograma da amostra do tubo de poliamida 12 (conforme recebido) com os picos cristalinos e halo amorfo ajustados.

Figura 4-24- Difratogramas das juntas soldadas de Poliamida 12 -

(a) Conjunto 1; (b) Conjunto 2; (c) Conjunto 3; (d) Conjunto 4; (e) Conjunto

5; (f) Conjunto 6.

Figura 4-25- Difratogramas corrigidos das juntas soldadas de Poliamida 12 - (a) Conjunto 1; (b) Conjunto 2; (c) Conjunto 3; (d) Conjunto 4;

(e) Conjunto 5; (f) Conjunto 6.

Figura 4-26- Difratograma de todas as amostras das juntas soldadas de acordo com os conjuntos de parâmetros. 


\section{Lista de tabelas}

Tabela 2-1 - Parâmetros de soldagem da PA 11 e 12 - Conforme DVS 2207 [3].

Tabela 2-2 - Configurações dos parâmetros de soldagem de PA 11 e

PA12, em função da espessura do tubo [3].

54

Tabela 3-1 - Propriedades da poliamida $12 \quad 66$

Tabela 3-2 - Configurações dos parâmetros de soldagem.

71

Tabela 3-3 - Dimensões dos corpos-de-prova de acordo com a norma

$\begin{array}{ll}\text { ASTM D638 [44]. } & 72\end{array}$

Tabela 4-1 - Condições de soldagem realizadas nos tubos de PA $12 . \quad 79$

Tabela 4-2 - Limite de resistência dos corpos de prova de Poliamida

12 - condição conforme recebido.

80

Tabela 4-3 - Limites de resistência dos corpos de prova de Poliamida 12 soldados, com rebarba.

Tabela 4-4 - Limite de resistência dos corpos de prova de Poliamida 12 soldados, sem rebarba.

Tabela 4-5 - Resultados dos ensaios de rigidez.

Tabela 4-6 - Temperatura e entalpia de fusão, e grau de cristalinidade da amostra do tubo de PA 12 conforme recebido.

Tabela 4-7 - Temperatura e entalpia de fusão, e grau de cristalinidade das amostras das juntas soldadas, de PA 12.

Tabela 4-8 - Temperatura e entalpia de fusão, e grau de cristalinidade 
das amostras das rebarbas das juntas soldadas, de PA 12.

Tabela 4-9 - Informações obtidas a partir das curvas TG-DTG das

amostras das juntas soldadas.

Tabela 4-10 - Informações obtidas a partir das curvas TG-DTG das

amostras das rebarbas das juntas soldadas.

Tabela 4-11 - Parâmetros de ajuste dos padrões de difração das amostras das juntas soldadas e do material conforme recebido, e os picos utilizados.

Tabela 4-12 - Dados obtidos através da difração de raios $x$ para todas as amostras soldadas e material conforme recebido. 


\section{Lista de Abreviaturas}

ABNT Associação Brasileira de Normas Técnicas

ASTM American Society for Testing and Materials

CEFET Centro Federal de Educação Tecnológica Celso Suckow da

Fonseca

CTS Centro de Tecnologia Senai

DE Diâmetro Externo Nominal

DEC Departamento de Engenharia Civil

DIN Deutsches Institut für Normung

DRX Difração de Raios $X$

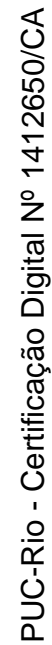

DSC Differential scanning calorimetry

DVS Deutscher Verband Fur Schweisstechnik

FTIR Fourier transform infrared spectroscopy

ISO International Organization for Standardization

LEM Laboratório de Estruturas e Materiais

LR Limite de Resistência

MPO Máxima Pressão de Operação

MS Mato Grosso do Sul

PA Poliamida

PN Pressão Nominal

PR Paraná

RS Rio Grande do Sul

UV Ultra Violeta 